\title{
REVIEW
}

\section{Scabies: more than just an irritation}

\author{
J S McCarthy, D J Kemp, S F Walton, B J Currie
}

Postgrad Med J 2004;80:382-387. doi: 10.1136/pgmj.2003.014563

Human scabies, caused by skin infestation with the arthropod mite, Sarcoptes scabiei, typically results in a papular, intensely pruritic eruption involving the interdigital spaces, and flexure creases. Recent research has led to a reassessment of the morbidity attributable to this parasite in endemic communities, particularly resulting from secondary skin sepsis and postinfective complications including glomerulonephritis. This has led to studies of the benefits of community based control programmes, and to concerns regarding the emergence of drug resistance when such strategies are employed. The renewed research interest into the biology of this infection has resulted in the application of molecular tools. This has established that canine and human scabies populations are genetically distinct, a finding with major implications for the formulation of public health control policies. Further research is needed to increase understanding of drug resistance, and to identify new drug targets and potential vaccine candidates.

See end of article for authors' affiliations

Correspondence to: Dr James S McCarthy, Queensland Institute of Medical Research, Herston Road, Herston, QĹD 4029, Australia; j.mccarthy@sph. uq.edu.au

Submitted

9 September 2003

Accepted

6 November 2003
S abies is caused by skin infestation with the mite Sarcoptes scabiei. Its common name, the "itch mite" derives from the severe pruritis that it causes. While the pruritis itself causes significant distress, breaks in the epidermis due to the burrowing of the mites, and the skin damage caused by the excoriation serve as portals of entry for pathogenic bacteria. The clinical consequences of secondary bacterial infection, especially with group A streptococci (Streptococcus pyogenes) result in significant and frequently unrecognised morbidity.

The global prevalence of this parasite is not known, but it has been estimated that despite the availability of effective treatments over 300 million people are infested. ${ }^{1}$ Scabies is well described in the historical record, with descriptions evident in ancient texts from China, India, and the Middle East. ${ }^{2}$

Like many other infectious diseases, it is transmitted by contact and therefore is a disease of overcrowding and poverty, but the role of poor hygiene is frequently overestimated. For example, in the Solomon Islands where the children spend much of their time in the sea the prevalence of scabies is very high among children living in overcrowded conditions. Thus, the stigma frequently associated with this and other ectoparasites such as head lice as markers of poor hygiene is misplaced. However, hygiene is thought to be important in the development and transmission of streptococcal skin sepsis following scabies.

\section{CLASSIFICATION}

$S$ scabiei is an arthropod, but only distantly related to insects. It is a member of the class Arachnida, subclass Acari (= Acarina), order Astigmata, and family Sarcoptidae. Sarcoptoid mites are slow moving obligate parasites that burrow into the skin of warm blooded animals including mammals (Sarcoptidae), and birds (Knemidokoptidae). The family Sarcoptidae, in addition to the human parasite $S$ scabiei, includes Notoedres cati, an ectoparasite of cats and Trixacarus caviae, an ectoparasite of guinea pigs. The $S$ scabiei adult female measures $0.4 \times$ $0.3 \mathrm{~mm}$, while the smaller adult male measures $0.2 \times 0.15 \mathrm{~mm}$.

The lifecycle of $S$ scabiei begins with the mating of adult male and female mites, after which the adult male dies, while the female begins to lay eggs. She continues to lay eggs in the skin burrow for her lifespan of 4-6 weeks, during which time she does not leave her burrow. Eggs are generally produced at a rate of $1-3$ eggs/day. After 3-4 days the eggs hatch, and six legged larvae cut through the roof of the burrow to reach the skin surface. They then dig burrows, and pass through two further developmental stages, protonymphs and tritonymphs, before moulting into either males or females. Less than $1 \%$ of the laid eggs develop to adult mites. ${ }^{3}$ All lifecycle stages can penetrate intact epidermis, achieving this by secreting enzymes that dissolve skin which is subsequently ingested. Skin entry can be achieved in less than 30 minutes.

During the first month of infection the mite population on an infected host increases, but at a rate far less rapidly than is theoretically possible, reaching a parasite burden of up to 25 adult females after 50 days, and up to 500 mites by 100 days. $^{3}$ However, the average burden on a normal host is 10-12 mites. Generally, after three months, mite numbers decrease rapidly. Likely explanations for the lower than possible rate of population growth include mechanical removal of the mites by scratching, as well as the host immune response. For example, sensitisation to mite antigens can be demonstrated a month after primary infestation. ${ }^{3}$ In experimental mite infestations of sheep, mite specific immunoglobulins and leucocytes attack mite mid-gut cells, and inhibit nutrient absorption. ${ }^{45}$

\section{SURVIVAL AND INFESTIVITY}

Human and canine scabies mites are capable of surviving for 24-36 hours at room temperature and retain the ability to re-establish infestation. Canine mites dislodged from a host respond to 
host odour and thermal stimuli by actively seeking their source. $^{6}$ Despite the detection, survival and penetrative abilities of $S$ scabiei when kept at room temperature, the role of contaminated bedding as a reservoir for infestation appears to be overemphasised. For example, in experiments where volunteers climbed into warm beds just vacated by heavily infected patients, only four new cases resulted from 272 attempts. $^{7}$

\section{CLINICAL MANIFESTATIONS}

Classical scabies in the adult presents as an intensely pruritic rash, particularly involving those regions of skin best suited for the mite. Onset of these symptoms in a host with no previous infestation occurs after 3-4 weeks. In adults and older children, sites of predilection include the interdigital web spaces, wrists, anterior axillary folds, periumbilical skin, pelvic girdle including buttocks, ankles, the penis in males, and the periareolar region in females. In infants and small children, and probably more commonly in both children and adults in tropical regions, the palms, soles, face, neck, and scalp may also be involved. Patients typically complain that the pruritis is more intense at night.

Two forms of skin eruption are typically observed: (1) erythematous papular or vesicular lesions that are associated with the burrows, and (2) a more generalised papular pruritic eruption unrelated to obvious mite activity. The burrow made by the adult female as it digests and consumes the epidermis is the classical diagnostic clinical sign of scabies. Burrows present as creeping (serpiginous) greyish lines, approximately $5 \mathrm{~mm}$ long; they are rarely visible with an unaided eye and are often absent. Egg cases and faecal pellets are found in the burrow. While the cause of the more generalised rash is yet to be conclusively established, it is most likely the result of the cellular immune response to mite antigens, as it takes 4-6 weeks to manifest in primary infestation. In subsequent infestations, sensitisation develops rapidly, and symptoms and signs are evident within 24-48 hours. This more generalised skin rash is most commonly seen around the axillae, the periareolar regions, abdomen, buttocks, and thighs.

\section{OTHER CUTANEOUS MANIFESTATIONS}

While the clinical picture in classical scabies should lead to ready diagnosis, infestation can result in a wide range of clinical appearances. Atypical appearances are most notable in patients with secondary infection (see below), and in those with longstanding infestation, where eczematous changes that range in severity from mild to severe may predominate. The appearance of scabies in patients taking steroids, topically as symptomatic treatment, or systemically may also be atypical, so-called scabies incognito. Likewise, the appearance may be difficult to recognise among those with immunosuppression either iatrogenic or due to advanced HIV infection or other immunosuppressive diseases.

\section{CRUSTED SCABIES}

Scabies is generally self limiting in humans but a small minority of people develop hyperinfestation, so-called crusted scabies, where the patient may harbour up to many millions of mites. This was formerly called Norwegian scabies on account of its first recognition in Norway in 1848 among patients with leprosy. ${ }^{8}$ There is no evidence of increased virulence of mites in such subjects; the aetiology is generally attributable to immunosuppression either iatrogenic or caused by specific diseases such as advanced HIV infection, lymphoma, malnutrition, etc. However, crusted scabies also has been reported among Indigenous Australians with no known immune deficiency. ${ }^{9-11}$ It has been speculated that such patients may have a specific immune deficit, the nature of which is yet to be defined.

The grossly thickened skin in crusted scabies shows the appearance of large hyperkeratotic warty crusts, typically involving the hands and feet but possible in all skin areas including trunk and scalp. These plaques are honeycombed with burrows that contain large numbers of mites, although burrows are rarely recognisable as such. Thick deposits of debris typically accumulate beneath the nails. The palms and soles may show deep fissuring of the crusts. These skin breaches along with other affected areas may show significant erythema, a sign of secondary infection, frequently with the typical skin pathogens Staphylococcus aureus and $S$ pyogenes. The presence of pruritis is variable. Generalised lymphadenopathy may be present, and a peripheral blood eosinophilia is common. Crusted scabies carries a high mortality, with deaths frequently occurring due to secondary sepsis. ${ }^{12} 13$

\section{SECONDARY INFECTION}

Untreated scabies is often associated with pyoderma, primarily caused by secondary invasion by $S$ pyogenes. ${ }^{14}$ Such infections can lead to cellulitis, boils, or lymphangitis. Streptococci and staphylococci have been isolated from skin burrows as well as from mite faecal pellets, suggesting that the mites themselves may contribute to the spread of pathogenic bacteria. Secondary infection of scabies with $S$ pyogenes is a major precipitant of acute post-streptococcal glomerulonephritis, ${ }^{15}{ }^{16}$ and possibly rheumatic fever. ${ }^{14}$ In community control programmes directed at scabies a significant reduction in the prevalence and severity of streptococcal pyoderma has been observed without concomitant use of antibiotics, reaffirming the importance of scabies as a major antecedent to streptococcal pyoderma. ${ }^{17}{ }^{18}$

\section{DIAGNOSIS}

A presumptive diagnosis can be made, based on a typical history of pruritis, worse at night, and the distribution of the inflammatory papules. A supportive history of contact with other cases can be frequently obtained. Parasitological confirmation of the diagnosis can be most easily obtained by gently scraping the skin off the burrow with a blunt scalpel blade, and placing the material on a glass slide with a drop of $10 \%$ potassium hydroxide or mineral oil, and seeking mites, eggs, or eggshells by low power microscopy.

\section{TREATMENT}

The primary means of treatment for scabies is by topical application of active substances, although oral treatment with ivermectin is an effective alternative. Sulphur compounds have been used for centuries with reasonable results, but now are largely only of historical interest. They can cause skin irritation, are messy and smelly, require repeated application, and are therefore poorly acceptable to patients.

Benzyl benzoate, an ester of benzoic acid and benzyl alcohol was initially isolated from natural products, so-called balsam of Peru, and has been used effectively for more than 60 years in a $25 \%$ solution (Ascabiol). While it is recommended to leave the lotion on the skin for 24 hours, benzyl benzoate is a rapidly acting agent, ${ }^{19}$ and though no controlled studies have been undertaken a shorter exposure it is likely to be effective. The main side effect of benzyl benzoate is skin irritation in the first minutes after application. The burning sensation is not uncommonly so painful that the lotion has to be washed off, although the discomfort usually rapidly decreases in severity after several minutes. Benzyl benzoate is in vitro one of the most active antiscabietic agents, ${ }^{19}$ and if tolerated shows an excellent cure rate. Contact dermatitis, though rare, has been described. ${ }^{20}$ While rare case reports of 
neurotoxicity have been reported in children, ${ }^{21}$ this side effect has not been conclusively established. When benzyl benzoate is used for treatment of children and sensitive adults it is generally recommended to be diluted-possibly reducing its efficacy. Crotamiton 10\% (Eurax) has been widely used on children due to its low toxicity profile. However, to attain a satisfactory response, multiple applications are usually required (for five days).

Gamma benzene hexachloride 1\% (lindane, Kwell, Quellada) is an organochlorine insecticide that, on its introduction showed a cure rate of $98 \%$, even after an application of only six hours' duration. It was successfully used for many years, but recent reports indicate the development of tolerance (see below). Furthermore, it can be systemically absorbed when applied topically, especially when applied to damaged skin. Systemic absorption is higher in infants and small children. Neurotoxicity (particularly seizures) has been documented in infants, children, and among those with widespread skin damage treated with lindane. ${ }^{22-24}$ In addition, like other organochloride pesticides, it has been associated with rare cases of idiosyncratic aplastic anaemia. ${ }^{25-27}$ On account of concern about these toxicities, it has been withdrawn from sale in many countries.

Permethrin 5\% (Lyclear), though more expensive than other agents, is now considered the treatment of choice in Australia, the UK, and the United States. It is a widely used synthetic pyrethroid insecticide whose parent compound was originally derived from chrysanthemums. It is well tolerated, has low toxicity, is poorly absorbed across the skin, and the small percentage that is absorbed is rapidly metabolised. A single overnight application is equally as effective as lindane. ${ }^{21}$

Ivermectin is a macrocyclic lactone antibiotic of the avermectin group, derived from the soil dwelling actinomycete Streptomyces avermectalis. It shows broad spectrum activity against both nematodes and arthropods. It has been widely used for treatment of sarcoptic mange in animals, and in humans is the drug of choice for treatment of the filarial worm Onchocerca volvulus (causing river blindness), and the intestinal nematode Strongyloides stercoralis. It also has been widely used for treatment of the other filarial infections, including Wuchereria bancrofti and Brugia malayi (both causes of elephantiasis). More recently it has been shown to be efficacious as an oral treatment in humans for both uncomplicated and crusted scabies. When administered orally in two doses separated by an interval of two weeks it results in a cure rate approaching $100 \%{ }^{28}$ The drug is particularly useful in patients with crusted scabies where three doses of $200 \mu \mathrm{g} / \mathrm{kg}$ given on days 1, 15, and 29 have proved successful. ${ }^{29}$ However, relapses have been documented and more severe cases may require additional doses. ${ }^{12}$

There is a wealth of experience accrued in the Onchocerciasis Control Program, documenting the safety of ivermectin in non-pregnant adults. While anecdotal reports exist documenting the inadvertent administration of the drug to pregnant women without adverse fetal outcome, experience in this group is limited, and the drug therefore is advised against in pregnancy. Likewise, there is a paucity of safety data for children $<5$ years of age. In a recent report where ivermectin was administered to a cohort of nursing home patients for control of a scabies outbreak, an increase in death rate was observed in the subsequent six months compared with age and sex matched controls. ${ }^{30}$ However, this report has been criticised for not matching the patients for dementia and other co-morbidities. ${ }^{31} 32$

As has been highlighted in a recently published systematic review undertaken as part of the Cochrane collaborative review process, ${ }^{21}$ there is a paucity of sufficiently powered, randomised controlled trials whereby different topical regi- mens have been compared to each other or to oral ivermectin. Further, the practical barriers to undertaking such studies render it unlikely that they will ever be undertaken. Thus, the recommendation of permethrin over malathion or lindane for topical treatment is based on inference from the collection of relatively small clinical trials. ${ }^{21}$ Likewise the relative efficacy of topical therapy compared to oral ivermectin remains to be rigorously defined.

To ensure reliable cure with a topical acaricide, it should be applied to the entire skin surface except the eyes. This is particularly important in small children and the elderly, among whom the infestation not infrequently involves the scalp. Patient information sheets are useful educational aids, explaining the treatment, warning against excessive use, and noting that symptoms such as itch may take several days or even longer to resolve, even after successful treatment. All family members and close physical contacts should be treated. Bed linen and clothing should be washed, but do not require further processing.

\section{PERSISTENT SYMPTOMS}

Many patients experience persistent symptoms for up to two weeks after curative treatment. This is likely due to the ongoing immune response to mite antigens. However, if symptoms persist beyond this period, a number of possible explanations should be considered (box 1). These include: incorrect initial diagnosis, incorrect application of the topical scabicide, poor penetration of the agent into scaly skin or hyperkeratotic fingernails, reinfection from untreated contacts or contaminated fomites, misdiagnosis of secondary eczema as treatment failure, contact dermatitis caused by the topical therapy, or finally drug resistant infection.

Resistance to lindane has been well documented..$^{33}$ Permethrin resistance is now widespread in human head lice, and recent in vitro acaricide efficacy studies indicate $S$ scabiei mites in northern Australia are becoming increasingly tolerant to permethrin. ${ }^{19}$ Very recent experience with the use of multiple doses of ivermectin to treat two patients with crusted scabies suggested the in vivo development of resistance. ${ }^{34}$ Careful monitoring for drug resistance (particularly to permethrin and ivermectin) by epidemiological assessments and in vitro testing, as well as research to understand the mechanism of resistance to permethrin and ivermectin will be required to ensure successful eradication of scabies in endemic areas. This is critical to help avert the scenario of globally increasing resistance and epidemics, a situation that has already developed for head lice.

Box 1: Causes of persistent symptoms after treatment for scabies

- Incorrect initial diagnosis.

- Ongoing hypersensitivity to mite antigen.

- Secondary eczema.

- Sensitisation to topical acaricide.

- Reinfection from untreated contacts or contaminated fomites.

- Treatment failure due to incorrect application of the acaricide.

- Treatment failure due to insufficient penetration of agent into hyperkeratotic skin or nails.

- Treatment failure due to drug resistance in the mite population. 


\section{PATIENTS WITH RECURRENT SCABIES}

The management of patients with sequential episodes of scabies, especially if associated with heavily crusted areas of skin can be problematic. Even those considered adequately treated are often swiftly reinfected in communities where infestation continues to circulate. While most recurrences are likely to be due to reinfection from untreated contacts, treatment protocols involving three fortnightly doses of ivermectin, a treatment that would be expected to completely eradicate the mites, appears to be inadequate in some cases. ${ }^{12}$ Genetic studies on mites collected from patients with recurrent scabies suggests that some of these cases are recrudescent infestations. ${ }^{35}$ While there is a paucity of published controlled clinical trials of the best course of action in this setting, we have had some success at the Royal Darwin Hospital using a protocol for such recalcitrant crusted scabies that includes up to seven doses of ivermectin (at least $200 \mu \mathrm{g} /$ $\mathrm{kg} /$ dose given on days $0,1,7,8,14,21$, and 28) together with topical benzyl benzoate (full body application initially every 2-3 days), and topical keratolytic creams.

\section{INSTITUTIONAL OUTBREAKS}

Scabies represents an important threat in institutional settings, particularly hospitals and nursing homes. Spread of infestation to staff is well documented. ${ }^{36}{ }^{37}$ Heavily infested patients with crusted scabies represent the greatest risk for transmission; such patients should be nursed in isolation, and contact precautions should be instituted, including the wearing of long sleeved gowns and gloves during all physical contact with the patients. Treatment of all patients, staff, and visitors to an involved ward is key to arresting such outbreaks. Ivermectin is an effective agent for the control of refractory epidemics. ${ }^{38}$ While the significance of the report of an adverse experience in one nursing home outbreak where ivermectin was deployed has been debated, the use of ivermectin in such settings should be cautiously approached.

\section{POVERTY, OVERCROWDING, AND POOR HYGIENE}

The relationship between scabies and relative levels of poverty, crowding, and hygiene within a household and a community is complex. Individual and population susceptibilities must also be considered, as must levels of exposure. In some Australian Aboriginal communities, factors including reduced socioeconomic status, inadequate medical facilities, and overcrowding contribute to high levels of endemic scabies. Recent reports have again documented an association between significant overcrowding (up to 30 people per household) and a prevalence of scabies approaching 50\%. ${ }^{39}$ Similar levels of endemic scabies have been observed in India. The high incidence of scabies in individual family groups indicates that transmission is likely mediated by close personal contact, such as sharing a bed. Sexual contact is a likely important means of transmission between adults.

Scabies has been considered to cycle within populations with a periodicity of greater than 10 years, suggesting that population based immunity may be important. However this explanation does not account for scabies that is endemic in many tropical and subtropical communities (for example, India, South Africa) without any apparent fluctuations in overall incidence. ${ }^{2} 4041$

Scabies affects people from all socioeconomic levels. Poverty and overcrowding, however, are often concomitant, and overcrowding is believed to encourage the spread of scabies. Scabies is not influenced by hygiene practices or the availability of water, as demonstrated by institutional outbreaks where high standards of hygiene exist, and by the experience among Kuna Indians, the indigenous population of Panama, a population in which careful daily personal hygiene is traditional. ${ }^{42}$
Community based treatment in communities where scabies is endemic has been repeatedly demonstrated to be the most effective means of control of scabies in such settings. In addition it results in a significant improvement in rates of secondary bacterial skin infection as well as postinfective complications such as glomerulonephritis. ${ }^{18} 43$

\section{ANIMAL SCABIES}

$S$ scabiei causes mange in many domestic and wild mammals worldwide, including dogs (S scabiei var canis), pigs, horses, camels, black bears, and monkeys (reviewed in Pence and Ueckermann $\left.{ }^{44}\right)$. In Australia, in addition to domestic animals and camels, sarcoptic mange has been recorded in the dingo (Canis dingo), the wild fox (Vulpes vulpes), and the common wombat (Vombatus ursinus). Extensive mortality in both foxes and wombats has been attributed to sarcoptic mange. Scabietic mange is a significant disease among some economically important livestock such as pigs. Current estimates indicate that between $50 \%$ and $95 \%$ of pig herds worldwide are infested with $S$ scabiei mites. ${ }^{45}$ Veterinary concerns include difficulties in diagnosis and control, and the economic effect of mange on feed conversion efficiency. In production herds the intense pruritus associated with the disease can interfere with milk production, weight gain, and leather quality and can inflict serious economic losses on primary industries. ${ }^{46}$

While reports exist of many of these animal infestations being transferred to humans, they differ clinically in a number of important respects. These include their atypical distribution on human skin compared with $S$ scabiei var hominis; the papules lack burrows, the incubation period is generally shorter, and the symptoms are generally transient in nature. Thus, if a patient is suspected of having contracted animal scabies, no treatment of the patient's human contacts is necessary. This approach is supported by experimental studies demonstrating only limited cross infestivity between different host species. $^{47}$ Further, molecular fingerprinting undertaken in over 700 mites from people and dogs in Australia and America, mite populations from communities where dog mange and human scabies are coendemic, support this hypothesis. Here, genotyping revealed that the mites segregate into two completely separate host associated populations (fig 1), ${ }^{35}$ and indicate that $S$ scabiei populations appear to be involved in separate transmission cycles.

\section{PROSPECTS FOR A VACCINE AGAINST SCABIES}

The observations that scabies infestation in individuals is self limiting, and that after the first infestation, significant protective immunity develops against reinfestation provide theoretical support for the development of a vaccine for scabies. ${ }^{78}{ }^{49}$ Further, the successful development and marketing of a vaccine for the cattle tick (Bm86, TickGARD ${ }^{50}$ ) supports the feasibility of this approach. Such a vaccine would have the potential to improve the quality of life of many poor people in overcrowded conditions worldwide. An effective vaccine would overcome the limitations of drug delivery and compliance, and emerging drug resistance. A vaccine against scabies mites would also be extremely useful in veterinary settings and commercial animal husbandry where infestation results in significant loss of productivity. While concerns exist regarding the possibility of generating a hypersensitivity reaction though vaccination, the cattle tick vaccine has passed rigorous safety evaluation. ${ }^{51}$

\section{CONCLUSION}

While the clinical manifestations of human scabies infestation have been well recognised for many years, a renewed appreciation of the role of this parasite in the causation of skin sepsis and subsequent postinfective complications has led to an augmentation of research on this much neglected 


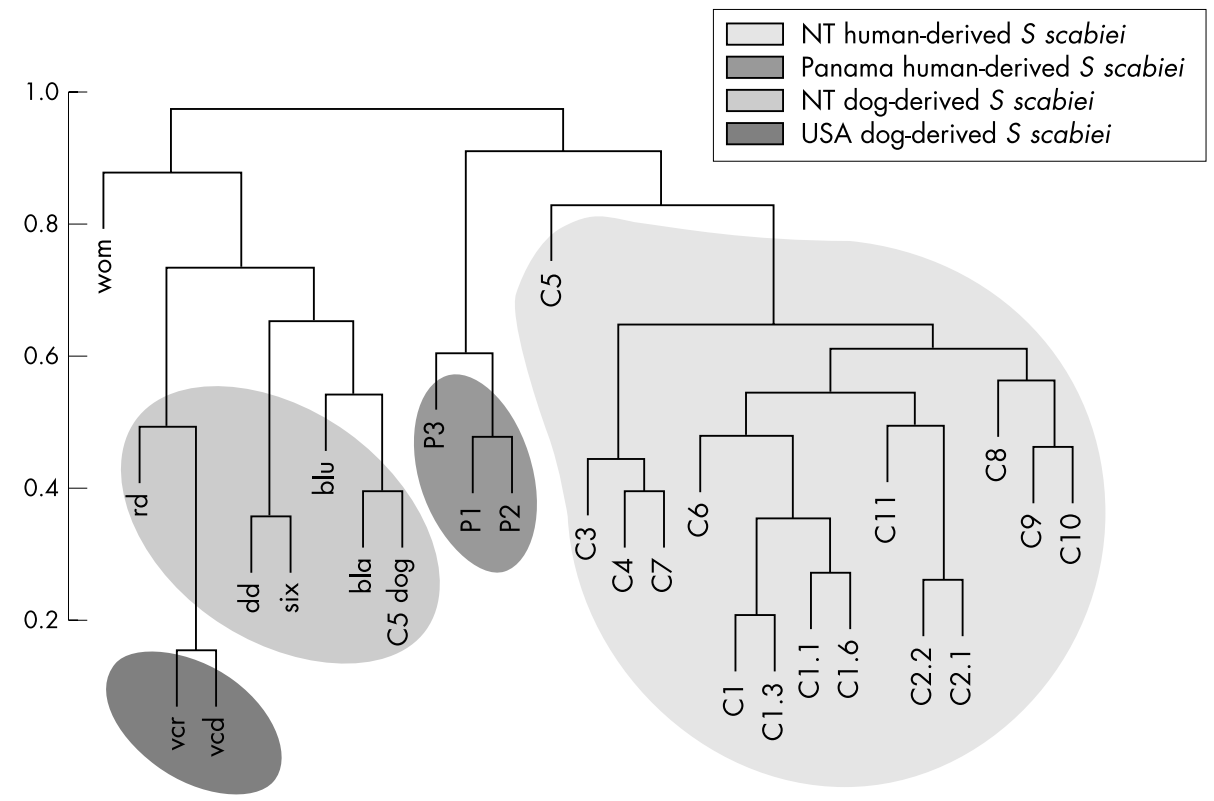

Figure 1 Relationships of mites from people and dogs. ${ }^{35}$ Irrespective of whether the mites were collected in Australia or America, mites from people segregate on one major branch of the dendrogram, while mites from dogs segregate onto the other. disease. Though the benefits of community based control programmes have been reproducibly demonstrated, the prospect of drug resistance and the gaps in knowledge of the relative efficacy and safety of various treatment options highlight the need for further research.

Significant recent advances in the study of the molecular biology of the scabies mite have already resulted in the clarification of the host specificity of strains of the parasite, and have enabled the separation of human and companion animal control programmes in areas where both are endemic. The new technologies offer the potential to improve our understanding of a wide range of other important issues. These include study of the biology of the mite, potentially leading to new therapies, study of the host immune response, leading to vaccine development, and study of the mechanisms of drug resistance leading to the development of tests to detect resistance. A further priority is further clinical studies on the relative safety and efficacy of current and new acaricides.

\section{ACKNOWLEDGEMENTS}

This work was supported by the Australian National Health and Medical Research Council, the Government of the Northern Territory of Australia, and by the Cooperative Research Centre for Aboriginal and Tropical Health. We would like to thank Dr Gregor Lawrence for his helpful comments.

\section{Authors' affiliations}

J S McCarthy, Queensland Institute of Medical Research, Brisbane, and Australian Centre for International and Tropical Health and Nutrition, University of Queensland, Brisbane, Australia

D J Kemp, Queensland Institute of Medical Research, Brisbane, Australian Centre for International and Tropical Health and Nutrition, University of Queensland, Brisbane, and Menzies School of Health Research, Darwin, and Co-operative Research Centre for Aboriginal Health, Australia

S F Walton, Menzies School of Health Research, Darwin, and Cooperative Research Centre for Aboriginal Health, Australia

B J Currie, Menzies School of Health Research, Darwin, and Cooperative Research Centre for Aboriginal Health and Northern Territory Clinical School, Flinders University, Royal Darwin Hospital, Australia

\section{REFERENCES}

1 Taplin D, Meinking TL, Chen JA, et al. Comparison of crotamiton 10\% cream (Eurax) and permethrin 5\% cream (Elimite) for the treatment of scabies in children. Pediatr Dermatol 1990;7:67-73.
2 Alexander JO. Scabies. Arthropods and human skin. New York: SpringerVerlag, 1984:227-92.

3 Mellanby K. Scabies in 1976. Royal Society of Health Journal 1977;97:32-6, 40.

4 Stromberg PC, Fisher WF. Dermatopathology and immunity in experimental Psoroptes ovis (Acari: Psoroptidae) infestation of naive and previously exposed Hereford cattle. Am J Vet Res 1986;47:1551-60.

5 Pettit D, Smith WD, Richardson J, et al. Localisation and characterisation of ovine immunglobulin within the sheep scab mite, Psoroptes ovis. Vet Parasitol 2000;89:231-9.

6 Arlian LG, Vyszenski-Moher DL, Pole MJ. Survival of adults and development stages of Sarcoptes scabiei var canis when off the host. Exp Appl Acarol 1989:6:181-7.

7 Mellanby K. The development of symptoms, parasitic infection and immunity in human scabies. Parasitology 1944;35:197-206.

8 Danielssen DC, Boeck W. Traite de la Spedalsked ou Elephantiasis des Grecs. Paris: J B Balliere, 1848.

9 Currie BJ, Maguire GP, Wood YK. Ivermectin and crusted (Norwegian) scabies. Med J Aust 1995;163:559-60.

10 Gogna NK, Lee KC, Howe DW. Norwegian scabies in Australian Aborigines. Med J Aust 1985;142:140-2.

11 Van Neste D, Lachapelle JM. Host-parasite relationships in hyperkeratotic (Norwegian) scabies: pathological and immunological findings. Br J Dermatol $1981 ; 105: 667-78$

12 Huffam SE, Currie BJ. Ivermectin for Sarcoptes scabiei hyperinfestation. Int J Infect Dis 1998:2:152-4

13 Hulbert TV, Larsen RA. Hyperkeratotic (Norwegian) scabies with gramnegative bacteremia as the initial presentation of AIDS. Clin Infect Dis 1992; 14:1 164-5.

14 Currie BJ, Carapetis JR. Skin infections and infestations in Aboriginal communities in northern Australia. Australas J Dermatol 2000:41:139-43.

15 Svartman M, Finklea JF, Earle DP, et al. Epidemic scabies and acute glomerulonephritis in Trinidad. Lancet 1972;i:249-51.

16 Hersch C. Acute glomerulonephritis due to skin disease, with special reference to scabies. S Afr Med J 1967:41:29-34.

17 Carapetis JR, Connors C, Yarmirr D, et al. Success of a scabies control program in an Australian aboriginal community. Pediatr Infect Dis $J$ 1997; 16:494-9.

18 Taplin D, Porcelain SL, Meinking TL, et al. Community control of scabies: a model based on use of permethrin cream. Lancet 1991;337:1016-8.

19 Walton SF, Myerscough MR, Currie BJ. Studies in vitro on the relative efficacy of current acaricides for Sarcoptes scabiei var hominis. Trans $R$ Soc Trop Med Hyg 2000;94:92-6.

20 Hogan DJ, Schachner L, Tanglertsampan C. Diagnosis and treatment of childhood scabies and pediculosis. Pediatr Clin North Am 1991;38:941-57.

21 Walker GJ, Johnstone PW. Interventions for treating scabies. Cochrane Database Syst Rev 2000;(3):CD000320.

22 Friedman SJ. Lindane neurotoxic reaction in nonbullous congenital ichthyosiform erythroderma. Arch Dermatol 1987; 123:1056-8.

23 Elgart ML. A risk-benefit assessment of agents used in the treatment of scabies. Drug Saf 1996;14:386-93.

24 Roos TC, Alam M, Roos S, et al. Pharmacotherapy of ectoparasitic infections. Drugs 2001;61:1067-88.

25 Rauch AE, Kowalsky SF, Lesar TS, et al. Lindane (Kwell)-induced aplastic anemia. Arch Intern Med 1990;150:2393-5.

26 Parent-Massin D, Thouvenot D, Rio B, et al. Lindane haematotoxicity confirmed by in vitro tests on human and rat progenitors. Hum Exp Toxicol $1994 ; 13: 103-6$. 
27 Albahary C, Dubrisay J Guerin Obstinate pancytopenia due to lindane (gamma isomer of hexachlorocyclohexane). Arch Mal Prof 1957; 18:687-91.

28 Elmogy M, Fayed H, Marzok H, et al. Oral ivermectin in the treatment of scabies. Int J Dermatol 1999:38:926-8.

29 Meinking TL, Taplin D, Hermida JL, et al. The treatment of scabies with ivermectin. N Engl J Med 1995;333:26-30.

30 Barkwell R, Shields S. Deaths associated with ivermectin treatment of scabies. Lancet 1997;349:1144-5.

31 Coyne PE, Addiss DG. Deaths associated with ivermectin for scabies. Lancet 1997;350:215-6; author reply 216.

32 Reinties R, Hoek C. Deaths associated with ivermectin for scabies. Lancet 1997;350:215; author reply 216

33 Boix V, Sanchez-Paya J, Portilla J, et al. Nosocomial outbreak of scabies clinically resistant to lindane. Infect Control Hosp Epidemiol 1997; 18:677.

34 Currie BJ, Harumal P, McKinnon M, et al. First documentation of in vivo and in vitro ivermectin resistance in Sarcoptes scabiei. Clin Infect Dis 2004 (in press).

35 Walton SF, McBroom J, Mathews J, et al. Crusted scabies: a molecular analysis of Sarcoptes scabiei var hominis populations in patients with repeated infestations. Clin Infect Dis 1999:29:1226-30.

36 Anonymous. Scabies in health-care facilities-lowa. MMWR Morb Mortal Wkly Rep 1988;37(11):178-9.

37 Degelau J. Scabies in long-term care facilities. Infect Control Hosp Epidemiol 1992;13:421-5.

38 Sullivan JR, Watt G, Barker B. Successful use of ivermectin in the treatment of endemic scabies in a nursing home. Australas J Dermatol 1997;38:137-40.

39 Currie BJ, Connors CM, Krause VL. Scabies programs in aboriginal communities. Med J Aust 1994;161:636-7.
40 Nair BK, Joseph A, Kandamuthan M. Epidemic scabies. Indian J Med Res 1977;65:513-8

41 Orkin M. Resurgence of scabies. JAMA 1971;217:593-7.

42 Taplin D, Rivera A, Walker JG, et al. A comparative trial of three treatment schedules for the eradication of scabies. J Am Acad Dermatol 1983:9:550-4.

43 Wong LC, Amega B, Connors C, et al. Outcome of an interventional program for scabies in an Indigenous community. Med J Aust 2001;175:367-70.

44 Pence D, Ueckermann E. Sarcoptic mange in wildlife. Rev Sci Tech 2002;21:385-98.

45 Cargill CF, Pointon AM, Davies PR, et al. Using slaughter inspections to evaluate sarcoptic mange infestation of finishing swine. Vet Parasitol 1997; 70(1-3):191-200.

46 Davis DP, Moon RD. Dynamics of swine mange: a critical review of the literature. J Med Entomol 1990;27:727-37.

47 Arlian LG. Biology, host relations, and epidemiology of Sarcoptes scabiei. Annu Rev Entomol 1989;34:139-61.

48 Arlian LG, Morgan MS, Vyszenski-Moher DL, et al. Sarcoptes scabiei: the circulating antibody response and induced immunity to scabies. Exp Parasitol 1994:78:37-50.

49 Arlian LG, Rapp CM, Morgan MS. Resistance and immune response in scabies-infested hosts immunized with Dermatophagoides mites. Am J Trop Med Hyg 1995;52:539-45.

50 Willadsen P. Novel vaccines for ectoparasites. Vet Parasitol 1997:71/23):209-22.

51 Bove O, Redondo M, Montero C, et al. Reproductive and safety assessment of vaccination with Gavac against the cattle tick (Boophilus microplus). Theriogenology 1999;51:1547-54.

\section{Call for papers}

10th European Forum on Quality Improvement in Health Care 13-15 April 2005, ExCel, Docklands, London For further information on how to submit your paper please go to: http://www.quality.bmipg.com 